\title{
LARGE-EDDY SIMULATION OF TURBULENT DIFFUSION WITH CHEMICAL REACTIONS IN THE CONVECTIVE BOUNDARY LAYER
}

\author{
UlRICH SCHUMANN \\ DLR, Institute of Atmospheric Physics, D-8031 Oberpfaffenhofen, F.R.G. \\ (First received 17 October 1988 and received for publication 30 January 1989)
}

\begin{abstract}
Bottom-up and top-down diffusion of two reacting air constituents in a dry homogeneous convective boundary layer with zero mean wind are simulated using large-eddy simulations. The model includes a simple binary, irreversible reaction without heat release. Subgrid-scale contributions of reactions are neglected as justified by spectral analysis of the results. The results depend upon the ratio $R$ of time scales for convection relative to that of the chemical reaction, on the concentration ratio of the two reacting components, and on the initial conditions. Cases with zero, finite and infinite reaction rate are considered. Without reactions, the bottom-up diffusivity is about twice as large as top-down diffusivity due to buoyancy effects. For infinite reaction rate, the thin reaction layer becomes highly convoluted. For $R>0.1$, i.e. for parameter values which are typical for the reaction between ozone and nitrogen oxide in the atmosphere, the reaction rate is strongly influenced by turbulence. The effective eddy diffusivities may be enhanced or reduced by chemical reactions, depending on the importance of gradient forcings of mass fluxes relative to other source and sink terms. A simple 'box-model' describes the time evolution of the mean concentrations in the mixed layer fairly well for $R \leqslant 0.1$ and for infinite reaction rate.
\end{abstract}

Key word index: Turbulent reacting flows, convective boundary layer, numerical simulation.

\section{INTRODUCTION}

The dependence of chemical reactions on turbulent mixing is of importance for many applications (Libby and Williams, 1980). For example, the reactions between air constituents like ozone $\left(\mathrm{O}_{3}\right)$ entrained from the stably stratified layers above the convective boundary layer (CBL) and nitrogen oxide (NO) emitted from dense traffic near the surface of the CBL is of importance with respect to air pollution problems (e.g. Seinfeld, 1986; Chang et al., 1987). For slow chemical reactions, turbulence succeeds in mixing the constituents before the chemical reactions become effective. Such situations can be described by so-called box-models. However, when the time scales of the chemical reaction are of the same order as, or much less than, the turbulent mixing time, which is often the case, the reaction rates are controlled by the ability of the turbulence to bring the chemical species together. For very rapid reactions between two components, the zone of reaction narrows, and in the limit, for an infinitely fast reaction, becomes a boundary surface between regions containing only one of the two reacting components. This is the "diffusive limit" which can be treated by means of the "conserved scalar approach" (O'Brien, 1971).

As summarized by Stull (1988) and Schmidt and Schumann (1989), turbulent motions in the CBL are asymmetric with strong narrow updrafts surrounded by large areas of slowly sinking motion. This structure was determined from large-eddy simulations (LES) first by Deardorff (1974). The updrafts are effective in transporting material from the surface upwards while components entrained from above the CBL are transported in the downdrafts at a slower rate. This asymmetry in bottom-up and top-down diffusion has been investigated using LES by Wyngaard and Brost (1984), Moeng and Wyngaard (1984) and Chatfield and Brost (1987) for passive, i.e. non-reacting and nonbuoyant components. Being aware of the fundamental limitations of the eddy-diffusivity concept as discussed by Lamb and Durran (1979), Wyngaard and Brost (1984) derived effective diffusivities for the bottom-up and top-down diffusion processes. More general first and second-order closure concepts are available (see Stull, 1988), but the values of the eddy diffusivities are still of interest with respect to pragmatic and simple turbulence modelling (Chang et al., 1987).

Riley et al. (1986) investigated the effects of turbulence on binary, irreversible reactions in a mixed layer driven by shear without buoyancy. Here, turbulence statistics are symmetric with respect to the shear plane. They integrated the Navier-Stokes equations together with balance equations for the fluid components to simulate directly the time-dependent and three-dimensional fields. This approach avoids subgrid-scale model-assumptions but is limited to moderate Reynolds numbers. Leonard and Hill (1988) used a similar approach to study mixing in homogeneous turbulence with two nonpremixed reactants. Herring and Wyngaard (1987) investigated a first-order chemically reactive scalar emitted at the surface of a con- 
vective layer at moderate Rayleigh numbers by direct numerical simulation. Pyle and Rogers (1980) discussed the transport of reactive trace gases by stationary planetary waves in the stratosphere. They show that eddy diffusivities are quite different for various species and depend on the time-scales for chemical and advection processes. Georgopoulos and Seinfeld (1986) review models for turbulent reacting plumes and introduce new closure models. We do not know of direct or large-eddy simulations of convective cases with binary reactions at large Rayleigh numbers.

In this paper, I.ES is applied to chemically reacting fluid constituents in a CBL. For this purpose, we use the LES method described in Schmidt and Schumann (1989) for a dry CBL over a homogeneous rough surface without mean wind, with turbulence driven by constant surface heat flux. For the non-reactive CBL, Schmidt and Schumann (1989) found very good agreement of the LES results with experimental observations employing fine resolutions $(160 \cdot 160 \cdot 48$ grid cells). For much coarser resolution $(40 \cdot 40 \cdot 20)$ the agreement with experimental results is still generally satisfying (Schmidt, 1988). The method is extended to include two scalar components which undergo binary, irreversible reactions at constant rate without heat release. The present study does not intend to be realistic in the chemical model; rather it is an exploratory study to demonstrate some basic processes. Thus, one cannot compare the concentration values with observations (e.g. Lenschow et al., 1980) because of the neglect of photochemical reactions. The realistic gas phase chemistry model used by Chang et al. (1987) comprises 77 reactions among 36 species. Such extensive models might be included in later LES at the expense of at least an order-of-magnitude larger computational effort.

The LES-method is described in section 2. In section 3 we repeat investigations of bottom-up and topdown diffusion of conservative tracers with new interpretations. The relevance of these results for infinite binary reaction rates is discussed in section 4 . Then, section 5 discusses the changes in the turbulent transport if the upward and downward transported components undergo binary reactions at finite rate. The importance of chemical reactions on the turbulent transport will be determined by comparing the effective diffusivities for cases without and with chemical reactions. The importance of turbulence on the chemical reaction will be assessed by comparing the actual amounts of the binary reaction rate with that computed from the mean values of the concentrations. At several places, we refer to a simple box-model described in the Appendix. The predictions of the boxmodel are tested by comparison to the LES in section 6 . Section 7 summarizes the results.

\section{THE LARGE-EDDY SIMULATION MODEL}

The numerical method MESOSCOP used in this paper is fully described in Schumann et al. (1987),
Schmidt (1988), Schmidt and Schumann (1989) and Elbert et al. (1989). Here, we review the essential features of the method and describe the treatment of chemical reactions.

The basic equations of the model describe the mass and momentum balances and the first law of thermodynamics in terms of grid-averaged velocities $u_{i}=(u, v$, $w$ ), temperature $T$ and two dynamically passive but chemically reacting fluid constituents $c_{m}, m=u, d$, (index $u$ for bottom-up and $d$ for top-down diffusing component $)$ as a function of the coordinates $x_{i}=(x, y$, $z$ ) and of time $t$. The Oberbeck-Boussinesq approximation is used; i.e. density $\rho$ is assumed to be constant except for buoyancy. The balance equations, written in Einstein's summation notation, arc:

$$
\begin{gathered}
\frac{\partial \bar{u}_{j}}{\partial x_{j}}=0 \\
\frac{\partial \bar{u}_{i}}{\partial t}+\frac{\partial\left(\bar{u}_{j} \bar{u}_{i}\right)}{\partial x_{j}}=-\frac{1}{\rho} \frac{\partial \bar{p}}{\partial x_{i}}+\frac{\partial}{\partial x_{j}}\left(v \frac{\partial \bar{u}_{i}}{\partial x_{j}}-\overline{u_{i}^{\prime \prime} u_{j}^{\prime \prime}}\right) \\
+\beta g \bar{T} \delta_{i 3}, \\
\frac{\partial \bar{T}}{\partial t}+\frac{\partial\left(\bar{u}_{j} \bar{T}\right)}{\partial x_{j}}=\frac{\partial}{\partial x_{j}}\left(\mu \frac{\partial \bar{T}}{\partial x_{j}}-\overline{u_{j}^{\prime \prime} T^{\prime \prime}}\right), \\
\frac{\partial \bar{c}_{m}}{\partial t}+\frac{\partial\left(\bar{u}_{j} \bar{c}_{m}\right)}{\partial x_{j}}=\frac{\partial}{\partial x_{j}}\left(\gamma \frac{\partial \bar{c}_{m}}{\partial x_{j}}-\overline{u_{j}^{\prime \prime} c_{m}^{\prime \prime}}\right) \\
.-\overline{c_{u} c_{d}}, \quad m=u, d .
\end{gathered}
$$

Here, $p$ is the pressure, $g$ is the gravitational acceleration, $\beta=-(\partial \rho / \partial T) / \rho$ is the volumetric expansion coefficient, $v, \mu$ and $\gamma$ are the constant molecular diffusivities of momentum, heat and concentrations (included here for completeness only), and $k$ is the reaction rate-coefficient of the binary chemical reaction. The bar denotes the average over a computational grid cell and the double-primes the deviations thereof. Angular brackets $<>$ will be used to denote mean values over a horizontal plane in the computational domain. They correspond approximately to ensemble mean values. Single primes denote fluctuations around such ensemble mean values.

The subgrid-scale (SGS) model and the model coefficients are the same as those described in Schmidt and Schumann (1989) and Ebert et al. (1989). The model uses the second-order algebraically approximated equations which account for the influence of buoyancy on subgrid-scale motions in addition to shear. However, we assume that SGS contributions from the chemical reactions are negligible. In particular, we approximate

$$
\overline{c_{u} c_{d}} \equiv \bar{c}_{u} \bar{c}_{d}+\overline{c_{u}^{\prime \prime} c_{d}^{\prime \prime}} \cong \bar{c}_{u} \bar{c}_{d} .
$$

Alternatively, we would have to find closures for the transport equations of $\overline{c_{u}^{\prime \prime} c_{d}^{\prime \prime}}, \overline{c_{m}^{\prime \prime 2}}, \overline{c_{m}^{\prime \prime} T^{\prime \prime}}$, and $\overline{c_{m}^{\prime \prime} u_{i}^{\prime \prime}}$, as given by Murthy (1975). For example, the first of this set of equations, for constant $k$ and equal diffusivities $\gamma$ 
for both components, reads as

$$
\begin{aligned}
\frac{\mathrm{D}}{\mathrm{D} t} \overline{c_{u}^{\prime \prime} c_{d}^{\prime \prime}} & +\frac{\partial}{\partial x_{j}}\left(\overline{u_{j}^{\prime \prime} c_{u}^{\prime \prime} c_{d}^{\prime \prime}}-\gamma \overline{\frac{\partial c_{u}^{\prime \prime} c_{d}^{\prime \prime}}{\partial x_{j}}}\right) \\
& =-\overline{c_{u}^{\prime \prime} u_{j}^{\prime \prime}} \frac{\partial \bar{c}_{d}}{\partial x_{j}}-\overline{c_{d}^{\prime \prime} u_{j}^{\prime \prime}} \frac{\partial \bar{c}_{u}}{\partial x_{j}}-2 \gamma \overline{\frac{\partial c_{u}^{\prime \prime} \partial c_{d}^{\prime \prime}}{\partial x_{j}}} \frac{\partial x_{j}}{} \\
& -k\left(\bar{c}_{d}\left(\overline{c_{u}^{\prime \prime 2}}+\overline{c_{u}^{\prime \prime} c_{d}^{\prime \prime}}\right)+\bar{c}_{u}\left(\overline{c_{d}^{\prime \prime 2}}+\overline{c_{u}^{\prime \prime} c_{d}^{\prime \prime}}\right)\right. \\
& \left.+\overline{c_{u}^{\prime \prime} c_{d}^{\prime \prime 2}}+\overline{c_{d}^{\prime \prime} c_{u}^{\prime \prime 2}}\right) .
\end{aligned}
$$

Here, D/D $t$ comprises the local plus the advective time derivatives. The time derivatives and the diffusive terms on the left of the equal sign are neglected in algebraical models. The terms on the right of the equal sign describe the generation of covariance due to fluxes times gradients minus diffusive and reactive destruction terms. The destruction terms require closure assumptions similar to those given for nonreactive turbulence by Launder (1975) and Schmidt and Schumann (1989). A complete SGS-model is obviously very complicated and closures of the reactive parts would introduce additional uncertainties. For these reasons, we neglect SGS contributions of chemical reactions and assume that $\overline{c_{u}^{\prime \prime} c_{d}^{\prime \prime}}$ is small in comparison to the resolved parts.

This assumption is valid if the grid spacing $\Delta$ is small in comparison to the integral length scale $\ell$ of turbulence because, by definition of the mean values of fluctuations, $\overline{c_{u}^{\prime \prime} c_{d}^{\prime \prime}} /\left(\bar{c}_{u} \bar{c}_{d}\right) \rightarrow 0$ for $\Delta / \ell \rightarrow 0$. The functional dependence of the cross-correlation on $\Delta$ is unknown, however, and hence it is not possible to quantify the error introduced by this assumption for finite grid spacing. Some estimate can be obtained by employing Schwarz' inequality, $\left(\overline{c_{u}^{\prime \prime} c_{d}^{\prime \prime}}\right)^{2} \leqslant \overline{c_{u}^{\prime \prime 2}} \overline{c_{d}^{\prime \prime 2}}$. It implies that the cross-correlation is small if the variances are small in comparison to the square of the grid-mean values. Moreover, for nonreactive cases, the variances may be estimated from the inertia theory of locally isotropic turbulence as summarized in Schmidt and Schumann (1989). This theory predicts the variance of concentrations to depend on the viscous dissipation $\varepsilon$ of turbulent kinetic energy, on the diffusive dissipation $\varepsilon_{m}$ of scalar variances, and the grid spacing $\Delta$ as $\left\langle\overline{c_{m}^{\prime \prime 2}}\right\rangle$ $=C\left\langle\varepsilon_{m}\right\rangle\langle\varepsilon\rangle^{-1 / 3} \Delta^{2 / 3}$, where $C$ is a constant of order unity. This estimate implies that the neglected crosscorrelation decreases in locally isotropic non-reacting turbulence at least as $\Delta^{2 / 3}$. Equation (6) shows that in the reacting case, the variances cause negative crosscorrelation but reactions reduce the cross-correlation if the latter becomes very large. These considerations show that it is very difficult to predict the size of the cross-correlations a priori.

Therefore, in section 5, we shall check the importance of the neglected cross-correlations a posteriori by investigating spectra of the covariances at resolved scales. Neglection of SGS covariances is justified if the cospectrum of $c_{u} c_{d}$ decreases with wave-number $k_{h}$ more quickly than $k_{h}^{-1}$.
The numerical integration scheme is based on an equidistant staggered grid and finite difference approximations. The momentum and continuity equations are approximated by second-order central differences in space which conserve mass, momentum and energy fairly well. Time integration is performed using the Adams-Bashforth scheme. The balance equations for temperature, concentration fields and for SGS kinetic energy are approximated by a second-order upwind-scheme. Pressure is computed by solving a discrete Poisson-equation employing fast Fouriertransform algorithms.

Chemical reactions are included in the model by updating $\bar{c}_{u}:=\bar{c}_{u}-S, \bar{c}_{d}:=\bar{c}_{d}-S$, after each integration step, where $S$ is the amount or reacting mass per time step,

$$
S=\frac{k \Delta t c_{u} c_{d}(1-\exp \{-D\})}{D+k \Delta t \min \left(c_{u}, c_{d}\right)(1-\exp \{-D\})}
$$

Here, $D \equiv \max \left(k \Delta t\left|c_{u}-c_{d}\right|, \varepsilon\right), \Delta t$ is the time step, and $\varepsilon$ a small number for which $1-\exp (-\varepsilon) \cong \varepsilon>0$. This procedure reproduces the exact solution for rapid reaction as given by O'Brien (1971). Otherwise the procedure is first order accurate, gives equal reaction rates for both components, and ensures positive and stable solutions for arbitrary reaction rate $k$ and arbitrary concentration ratio $c_{u} / c_{d}$.

Boundary conditions are periodic at the lateral boundaries of the cubical computational domain. At the top, free-slip boundary conditions are used, including a radiative condition for pressure. At the bottom, the heat flux $Q_{s}$ determines the SGS flux at this surface, and the vertical fluxes of horizontal momentum are evaluated from the Monin-Obukhov relationships. For the upward diffusing component, $\bar{c}_{u}$, the emission flux $E$ is prescribed as a constant at the surface. For the downward diffusing component, $\bar{c}_{d}$, the surface concentration is assumed to be zero and the corresponding flux is computed from the Monin-Obukhov relationships. Thus, the deposition flux of $\overline{\mathcal{C}}_{d}$ depends upon its concentration in the mixed layer.

The initial conditions for temperature, velocity and SGS energy are the same as those given in Schmidt and Schumann (1989). The initial temperature profile represents a constant-temperature mixed layer topped by a layer of uniform and constant stability. Small random temperature and velocity perturbations in the mixed layer initiate the convective motion. All fields are made non-dimensional in terms of a reference inversion height $z_{i 0}$, and the corresponding convective velocity $w_{*}$, and temperature $T_{*}$ scales (Stull, 1988, p. 118). The inversion height $z_{j}$ is determined in the simulations as that height where the vertical heat flux assumes its (negative) minimum. The specific inversion height $z_{i 0}$ selected for normalization is that obtained after six non-dimensional time-units $z_{i 0} / w_{*}$. At this time a quasi-steady state of turbulence has been reached. The upward diffusing component is set to zero initially. The downward diffusing component is 
set to $c_{d}^{*}$ above $z_{i 0}$ and set to $c_{d}^{0}$ below this height initially. In most cases, $c_{d}^{0}=0.4 c_{d}^{*}$; one case is run with $c_{d}^{0}=0$. As is shown in the Appendix, steady-state will not be reached with respect to the concentration values during a day. Therefore, the results are sensitive to the initial concentration values as will be discussed below.

The resultant CBL is characterized by a 'convective Froude number' $F r=w_{*} /\left(z_{i 0} N\right)=0.0922$, where $N$ is the Brunt-Väisälä frequency of the stable layer above the CBL. The surface roughness is set at $z_{0}=10^{-4} z_{i 0}$. Mean molecular transports are effectively zero. The scales of the concentration fields are $c_{u}^{*}=E / w_{*}$ for the upward diffusing component with prescribed emission flux $E$, and $c_{d}^{*}$ for the downward diffusing component. Parameters controlling the chemical reaction are the non-dimensional reaction rate $R$ and the concentration ratio $n$ :

$$
R=k z_{i 0} \frac{c_{u}^{*}}{w_{*}}, n=\frac{c_{d}^{*}}{c_{u}^{*}} .
$$

Several cases with various values of these parameters are considered: $R=0,0.1,1,2, \infty ; n=1,20,40$. The values $R=1, n=20$ arise for example if $w_{*}=1.46 \mathrm{~m} \mathrm{~s}^{-1}, z_{i 0}=1600 \mathrm{~m}, E=7.4 \times 10^{16} \mathrm{~m}^{-2} \mathrm{~s}^{-1}$, $c_{d}^{*}=1.014 \times 10^{18} \mathrm{~m}^{-3}$, for which $c_{u}^{*}=5.07 \times 10^{16} \mathrm{~m}^{-3}$. These parameters are typical for NO diffusing upwards and $\mathrm{O}_{3}$ being entrained from above the CBL. We assume that these constituents undergo the model reaction

$$
\left[\mathrm{O}_{3}\right]+[\mathrm{NO}] \rightarrow\left[\mathrm{NO}_{2}\right]+\left[\mathrm{O}_{2}\right]
$$

with the rate constant $k=1.8 \times 10^{-20} \mathrm{~m}^{3} \mathrm{~s}^{-1}$ as given for $T \cong 300 \mathrm{~K}$ by Seinfeld (1986), p. 175. Of course, this is a simplistic model of air chemistry used for illustration purposes only.

The computational domain extends horizontally over a domain of size $5 z_{i 0}$ in $x$ and $y$-directions, and vertically from height $z=0$ to $z=1.5 z_{i 0}$. The number of grid cells was varied. Most parameter studies are performed for 'grid C' (coarse) with $40 \cdot 40 \cdot 24$ cells, where the last number counts the grid cells in the vertical direction. These simulations for $0 \leqslant t w_{*} / z_{i 0} \leqslant 8$ require $3300 \mathrm{~s}$ of computing time on a CRAY-XMP. The reference cases $(n=20, R=0$ and 1) are also investigated for 'grid $M$ ' (medium) with $80 \cdot 80 \cdot 24$ cells $(10,900 \mathrm{~s}$ compuling time). The fine grid with $160 \cdot 160 \cdot 48$ cells, which was used by Schmidt and Schumann (1989) and Ebert et al. (1989), is not applied in this exploratory study because of excessive computing time ( $36 \mathrm{~h}$ for the present problem). The time step is set to $\Delta t=0.004 z_{i 0} / w_{*}$ for grids $C$ and $M$.

\section{BOTTOM-UP AND TOP-DOWN DIFFUSION WITHOUT CHEMICAL REACTIONS}

Figure 1 shows a vertical cross-section through the computational domain with contours of the vertical velocity and the concentrations of the two tracers for $R=0$, i.e. without chemical reactions. The results are obtained for the grid $M$ at time $t_{*}=t w_{*} / z_{i 0}=5$. The vertical velocity exhibits several strong and rather isolated updrafts surrounded by large downdraft
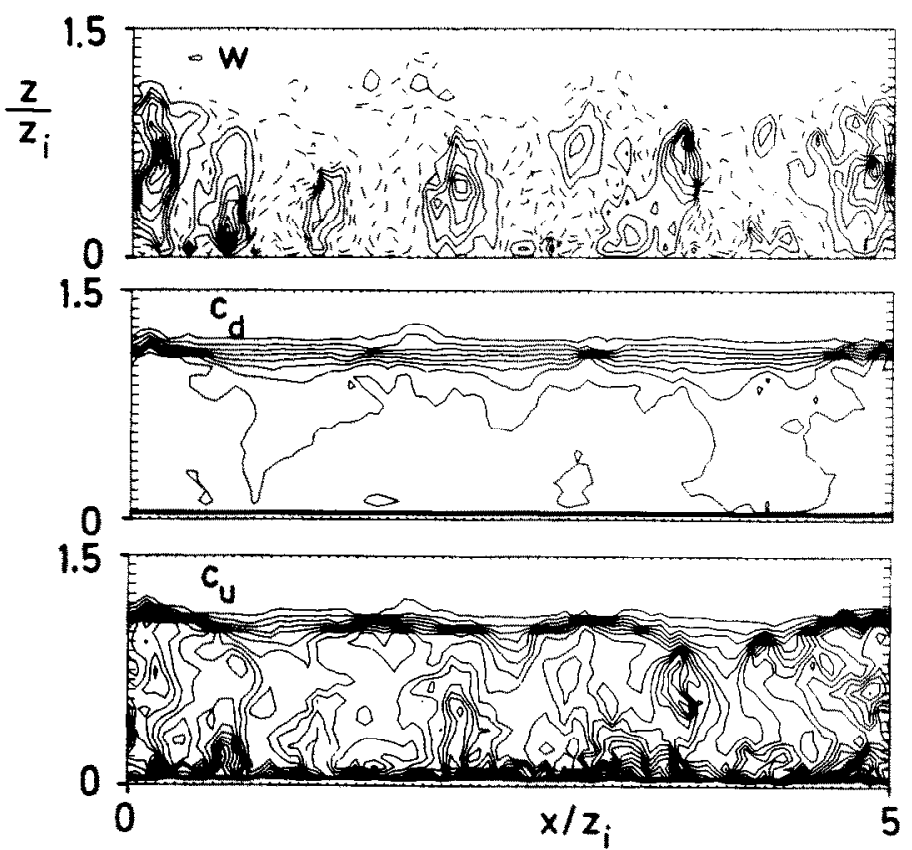

Fig. 1. Vertical velocity $\bar{w}$, top-down concentration $\bar{c}_{d}$, and bottom-up concentration $\bar{c}_{w}$ at time $t_{*} \equiv t w_{*} / z_{i}=5$ in a vertical cross-section without chemical reactions. The contour increments are $0.3 w_{*}, 0.08 c_{d}^{*}, 0.6 c_{u}^{*}$, for $w$, $\bar{c}_{d}, \bar{c}_{u}$, respectively. Full curves: positive contour values; dashed curves: negative values. 
areas. The concentration field of the upward diffusing component is large near the surface where this component is fed into the surface layer at constant rate. In the surface layer, this component is advected first towards the bases of updrafts and then carried upwards within the updrafts. Near the inversion the vertical diffusion is much reduced because of the stability of the inversion layer. At this height, the upward motions are deflected sidewards into downdrafts which fill up the mixed layer with this component. The opposite process occurs with the downward diffusing component. It is entrained at a small rate from above the inversion between the penetrating updrafts, and the maximum concentration values inside the mixed layer occur within the downdrafts.

The actual fraction of horizontal area containing upward and downward motion in terms of vertical velocity is plotted vs height in Fig. 2 together with the mean velocities in the updraft and downdraft areas. Figure 2 shows that the area containing upward and downward motions are about the same near the surface and above the inversion. The updraft area fraction in the mixed layer is less than the downdraft area. This is the effect of buoyancy, which causes updrafts to accelerate while rising with reduced crosssection for continuity. Downdrafts sink only because of pressure forces which drive air masses downwards to replace rising fluid for continuity. Hence, they are decelerating and occupy larger area fractions. The area fraction of updrafts is smallest in the upper portion of the mixed layer because only a few but very strong updrafts succeed in overcoming the weakly stable stratification in the upper half of the mixed layer. An updraft area-fraction $<0.5$ implies positive skewness of the vertical velocity (Wyngaard, 1987). Since the skewness is positive everywhere in the mixed layer, the updraft area-fraction should not exceed a value of 0.5 . In contrast Chatfield and Brost (1987) find an area fraction of updrafts $>0.5$ near the surface.
Schmidt and Schumann (1989) discuss various reasons to explain the appearance of such unrealistic negative skewness values.

The mean concentration profiles at a sequence of times are plotted in Fig. 3. The upward component increases at an approximately constant rate because all material introduced at the surface is distributed nearly evenly over the mixed layer with very little loss to the stable layer above the inversion. This loss is actually caused by dilution with clean air entrained from above the inversion. But certainly also numerical diffusion contributes to the loss because of the relatively coarse resolution of this stable layer. Only after a time of order $\mathrm{Fr}^{-2} z_{i} / w_{*}$ (see Appendix), a quasi steady state can be expected in which the surface flux is balanced by the dilution. For $c_{d}^{0}=0.4 c_{d}^{*}$, the profile of the downward diffusing component reaches such a steady state much earlier when the amount of entrained mass equals approximately the mass flux to the surface. This mass flux can be estimated as explained in the Appendix.

Figure 4 shows the effective eddy diffusivities computed from the concentration mean profiles and the LES results for the vertical mass fluxes. The results exhibit some statistical sampling uncertainty becausc the mean profiles are based on a horizontal mean value within the limited-size computational domain. Occasionally, the vertical gradients of the mean profiles change their sign, so that infinite and negative eddy diffusivities are computed. The sign change can be spurious due to the statistical uncertainties but also physical because of local maxima created near the inversion by strong upward transport in updrafts. These reasons explain the large scatter and the appearance of infinite values of the diffusivities. Some of the persistent oscillations in the lower quarter of the mixed layer might also be caused by finite-difference approximations. The mean values (dotted curves) evaluated from time-averaged concentration profiles
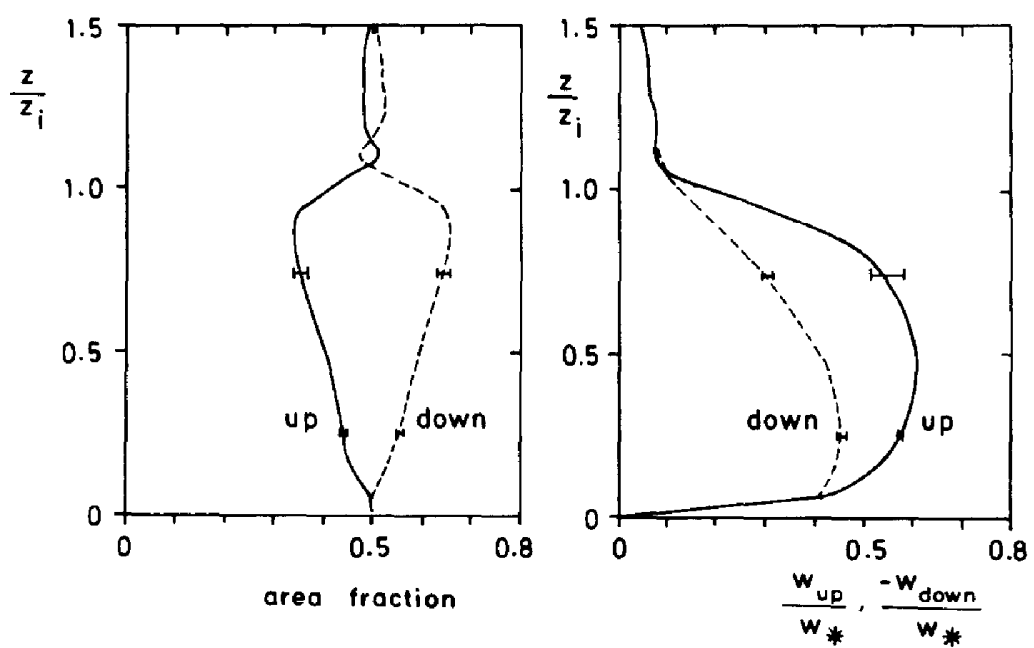

Fig. 2. Area fractions of upward and downward motions and mean values of the vertical velocities in the two areas. 


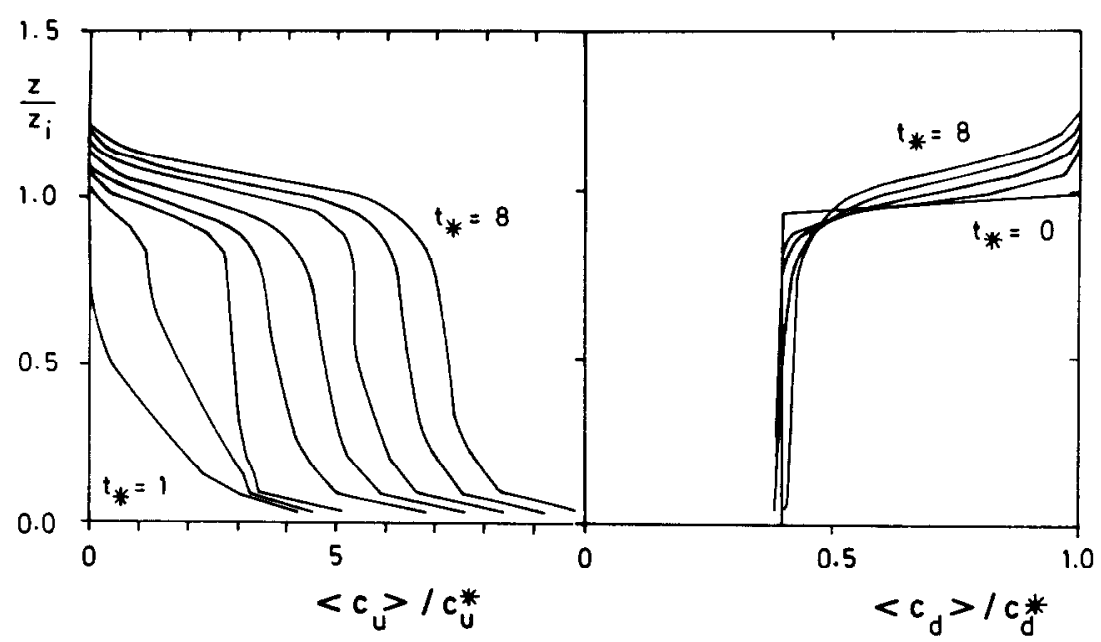

Fig. 3. Mean concentration profiles vs normalized height at a sequence of non-dimensional times for top-down and bottom-up diffusing component (without reactions).

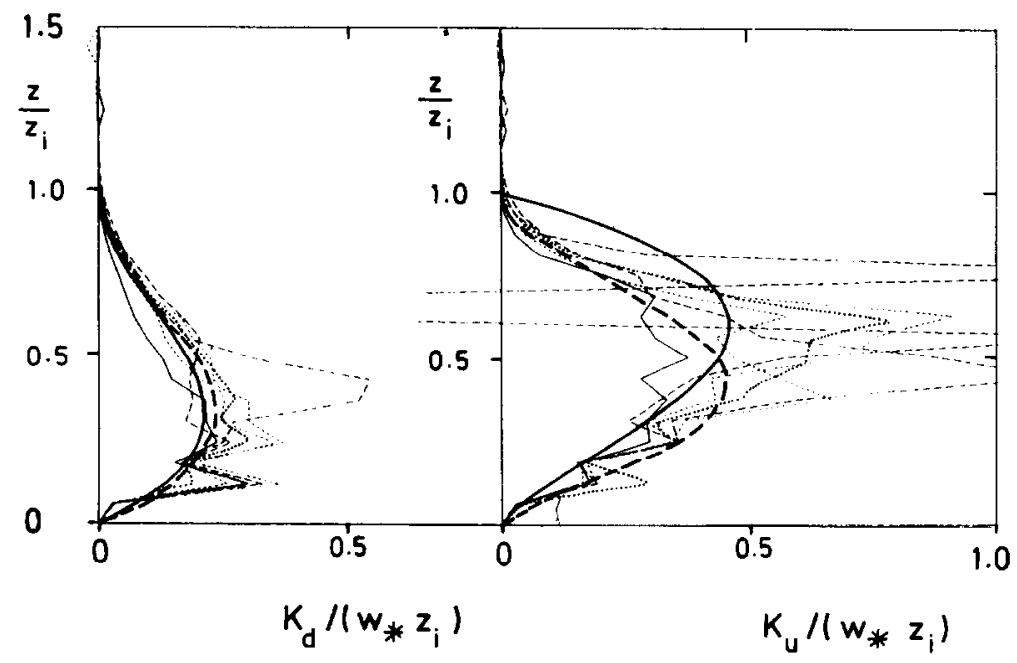

Fig. 4. Effective eddy-diffusivities $K_{u}$ and $K_{d}$ vs height at a sequence of nondimensional times. Dotted curve: mean diffusivities; thick full curves: interpolations from Moeng and Wyngaard (1984); dashed curves: Equation (10).

and fluxes in the period $5 \leqslant t_{*} \leqslant 8$ show clearly, however, that the eddy diffusivity for bottom-up. diffusion is much larger than that for top-down diffusion. Similar results have been reported by Wyngaard and Brost (1984), Moeng and Wyngaard (1984) and Wyngaard (1987). The full curves in Fig. 4 are the interpolation curves suggested by Moeng and Wyngaard (1984). Our results show rather small values of $K_{u}$ in the upper part of the mixed layer and are better fitted by the approximations

$$
\begin{aligned}
& \frac{K_{u}}{w_{*} z_{i}}=5.0\left(\frac{z}{z_{i}}\right)^{3 / 2}\left(1-\frac{z}{z_{i}}\right)^{2}, \\
& \frac{K_{d}}{w_{*} z_{i}}=1.6\left(\frac{z}{z_{i}}\right)\left(1-\frac{z}{z_{i}}\right)^{2} .
\end{aligned}
$$

In any case, the bottom-up diffusivity is about twice as large as the top-down diffusivity. Wyngaard (1987) explains this fact by the asymmetric structure of the CBL together with the time-dependence of the increasing bottom-up diffusion component. As an alternative we offer the following explanation. The turbulent transport is large if the corresponding velocity is large and if the flow is from places with high concentrations to places with low concentration. The upward diffusing component is gathered from the surface into the updrafts. These updrafts are buoyant and accelerate upwards. Thus they transport material upwards in an effective manner. In contrast, downward diffusing components are entrained from above the inversion into downdrafts. These downdrafts contain fluid from the relatively warm layer above the inversion and thus 
do not tend to sink from buoyancy forces. In fact, as can be seen from Fig. 2, the downdraft velocity is rather small near the inversion. Moreover, the downdrafts incorporate fluid coming from updrafts which do not reach the inversion but dilute the concentration $c_{d}$ in the downdrafts. Hence, downdrafts are much less effective in transporting fluid components.

Formally, this difference can be explained by reference to the transport equations for the ensemble-mean vertical fluxes which are similar in structure to Equation (6). Neglecting diffusional contributions and abbreviating sink terms due to pressure-concentration correlations and diffusive dissipation by $\Psi$, these transport equations for zero mean wind in the nonreactive case reduce to

$$
\begin{aligned}
\frac{\mathrm{D}}{\mathrm{D} t}\left\langle c_{m}^{\prime} w^{\prime}\right\rangle= & -\left\langle w^{\prime} w^{\prime}\right\rangle \frac{\partial\left\langle c_{m}\right\rangle}{\partial z} \\
& +\beta g\left\langle c_{m}^{\prime} T^{\prime}\right\rangle-\Psi, m=u, d .
\end{aligned}
$$

We see that the vertical mass flux of each component is driven by the vertical gradient of mean concentration and the concentration-temperature correlation under gravity. The latter increases the flux for bottom-up diffusion particularly near the surface where mass and heat are emitted at comparable rate resulting in $100 \%$ correlation at the surface. In contrast, top-down diffusion becomes reduced by buoyancy because entrained fluid is warm and rich in material $c_{d}$ in the interfacial layer resulting in a positive correlation which reduces the downward flux magnitude. The importance of buoyancy contributions has been stressed also by Sun (1986). Thus the transient aspect used by Wyngaard (1987) for explanation of the difference between the two types of diffusivities might be of second order importance only.

\section{BOTTOM-UP AND TOP-DOWN DIFFUSION WITH VERY RAPID CHEMICAL REACTIONS}

For very large reaction rate-coefficients, chemical reactions quickly consume the component which has the smaller concentration initially. As a result, two regions are formed, each containing only one of the two components. Thereafter, reactions take place only at the interface between these two regions and the reaction rate is completely controlled by the amount of material diffused towards this interface. As shown in O'Brien (1971), in this 'diffusive limit' the concentrations are determined by the solution for a non-reacting scalar $c=c_{u}-c_{d}$ with corresponding initial and boundary conditions. The solutions after depletion of the smaller concentration component are

$$
\begin{gathered}
c_{u}=c_{,} c_{d}=0, \text { for } c>0, \\
c_{d}=-c, c_{u}=0, \text { for } c<0 .
\end{gathered}
$$

Thus, the infinite reaction case can be diagnostically determined from the simulations without chemical reaction. For example, Fig. 5 shows the concentration field of the upward diffusing component at a certain instant of time, both for cases with finite and zero initial concentration $c_{d}^{0}$ in the mixed layer. The reaction takes place at the zero contour line of this field. We see that the reaction interface is strongly convoluted. The component $c_{w}$ is carried with the updrafts and single spikes of updrafts reach the inversion. Occasionally, small isolated blobs of fluid containing the emitted component occur as remainders of previous updrafts. For $c_{d}^{0}=0$, the emitted quantity fills up the mixed layer quickly and the reaction interface is inverted downwards at places with strong downdrafts. As a consequence, the mean profiles of the two components (see Fig. 6) indicate an overlapping region where

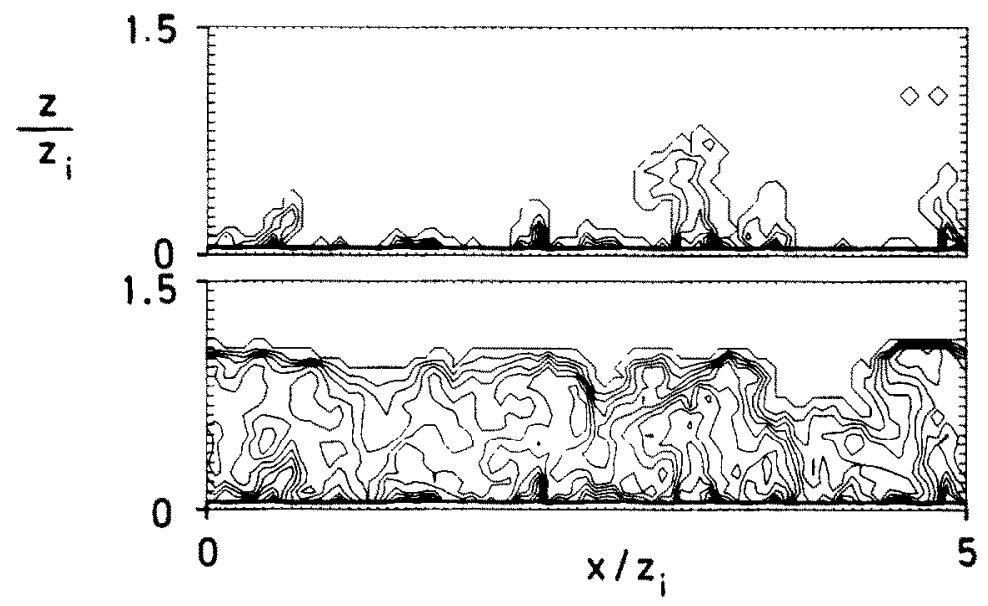

Fig. 5. Bottom-up concentration $\bar{c}_{u}$ at time $t_{*}=6$ in a vertical cross-section for infinite reaction rate. The contour increment is $1.0 c_{w}^{*}$. Upper panel: $c_{d}^{0}=0.4 c_{d}^{*}$; lower panel: $c_{d}^{0}=0$. 


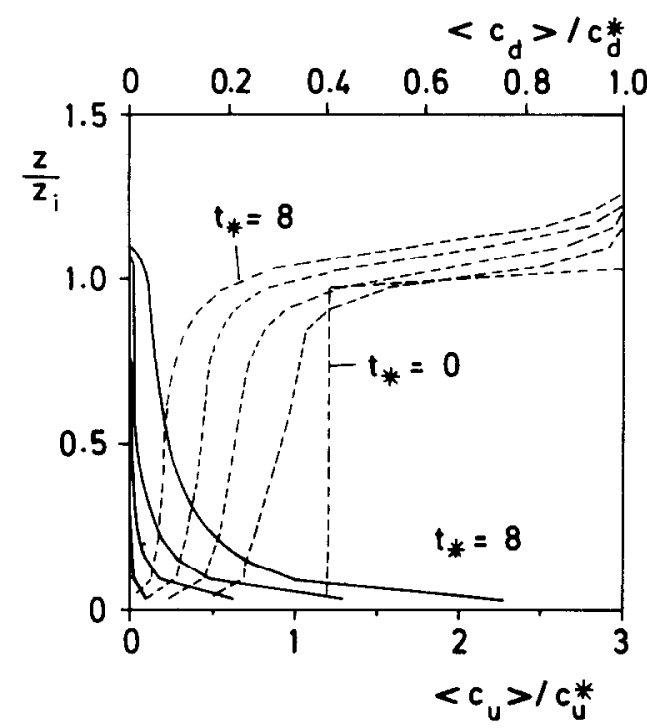

Fig. 6. Mean concentration profiles of $c_{w}$ (full curves) and $c_{d}$ (dashed) vs normalized height at a sequence of non-dimensional times for top-down and bottom-up diffusing components for $c_{d}^{0}=0.4 c_{d}^{*}$ with infinite reaction rate.

both components coexist (at different lateral positions) in spite of the infinite reaction rate. This overlap spans practically the whole CBL. Such results are very difficult to determine from one-dimensional models. Similar results for the mixed shear-layer have been reported by Riley et al. (1985) from direct simulations.

Without further simulations, these results and the box model allow us to deduce some general conclusions on the effect of turbulence on the chemical components. The vertical height and extension of the reaction zone depends on the initial values and the ratio of fluxes of materials from bottom-up and topdown. For small initial concentrations of $c_{d}$ in the mixed layer of the $\mathrm{CBL}$, the reaction interface approaches the top of the mixed layer. Also, for smaller values of the concentration ratio $n=c_{d}^{*} / c_{u}^{*}$, the amount of material entrained from above the CBL is smaller resulting in a larger mean height of the reaction zone. Conversely, for smaller emission rates of the bottom-up component or larger initial values of the top-down concentration, the reaction zone approaches the lower boundary of the CBL. The reaction zones are narrow and either close to the surface or close to the inversion layer if one of the components dominates. At late times, when the influence of the initial values is small, the reaction zone depends on the relative amounts of vertical fluxes of the two components. If the amount of emission of $c_{u}$ exceeds the amount of entrainment of material $c_{d}$ from above, then the average reaction zone extends over the whole mixed layer of the CBL. For steady state, the vertically integrated reaction rate by which both materials are depleted is independent of the actual value of the (large) reaction rate coefficient. It equals the emission rate of bottom-up diffusing component or the rate at which material is entrained from above the CBL, whichever gives the smaller rate. Hence, the reaction rate is totally different from the product of the mean values and the infinite reaction rate-coefficient.

\section{BOTTOM-UP AND TOP-DOWN DIFFUSION WITH CHEMICAL REACTIONS AT FINITE RATE}

In this section, results for finite chemical reaction rates, $0.1 \leqslant R \leqslant 2$, are reported. First, we discuss a reference case with $R=1, n=20$, and various initial values $c_{d}^{0}$ of $c_{d}$ in the mixed layer below the inversion. Thereafter, we will report on results where $R$ or $n$ are varied.

\section{Reference cases}

Figure 7 shows results for $R=1, n=20$. The left panels were obtained for the same flow field as those shown in Fig. 1, i.e. with finite initial value of the down-diffusing component in the mixed layer. Subsequently we call this the 'mixed case' because it assumes that some of the upper component has been mixed downwards before the reaction starts. The right panels apply to $c_{d}^{0}=0$, which is the 'unmixed case'. One observes from Fig. 7 approximately the same updraft and downdraft structure as in Fig. 1 but the concentration values inside the mixed layer are reduced due to the chemical reactions. The reaction takes place in those regions where both components are present to sufficiently high degrees. In the mixed case, this region is closer to the bottom surface than in the unmixed case. This was to be expected from what we have learned for the infinite reaction case. However, the reaction zone in this simulation is of considerable width and certainly well resolved by the LES (grid M). One expects that this width decreases with increasing value of $R$ so that the diffusive limit is approached for about $R>10$. One might expect that the reaction zone appears mainly in what Stull (1988), p. 465, calls the "intromission zone" between updrafts and downdrafts, i.e. the regions where updrafts and downdrafts are mixing laterally. However, due to the strong dependence on the concentration ratio, the reactions maximize inside updrafts and in the surface layer. In the unmixed case, considerable reactions also occur at the inversion.

Figure 8 shows the mean profiles both for the mixed and unmixed cases as evaluated from grids $\mathrm{C}$ and $\mathrm{M}$. The differences between the results from the two grids are not small but do not affect the qualitative trends. Subsequently, all results shown are from grid $\mathrm{C}$. The results show, that the downward transported component is quickly reduced by chemical reactions in the mixed layer. The upward transported component cannot accumulate in the mixed case because a nondimensional time of order $0.4 n=8$ is required before the initial values of $c_{d}$ are depleted by reactions with the component emitted from the surface. By contrast, 

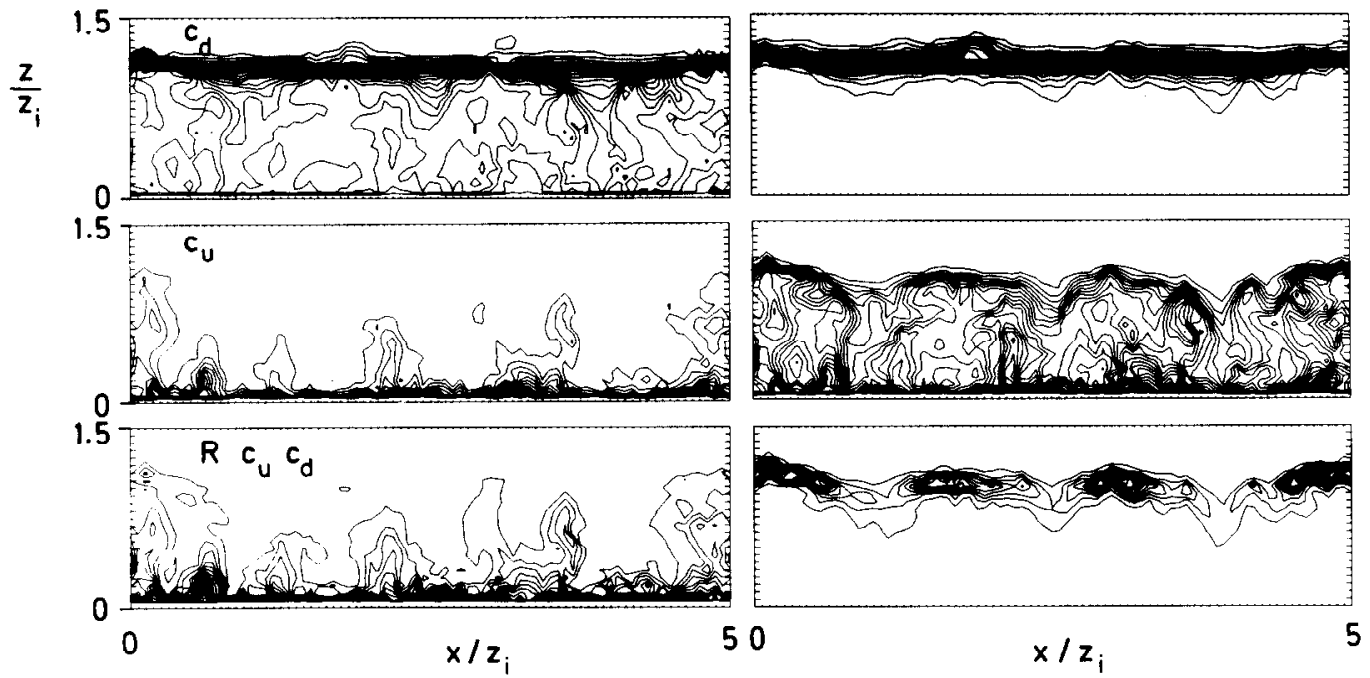

Fig. 7. Concentrations $\bar{c}_{d}, \bar{c}_{\mu}$ and reaction rate $R \bar{c}_{u} \bar{c}_{d}$ in a vertical cross-section as in Fig. 1 but with chemical reactions $(R=1, n=20)$. Left panels: $c_{d}^{0}=0.4 c_{d}^{*}$; right panels: $c_{d}^{0}=0$. Contour line increments: $0.05 c_{d}^{*}, 0.5 c_{u}^{*}$, $0.025 c_{u}^{*} c_{d}^{*}$, from top to bottom, respectively.
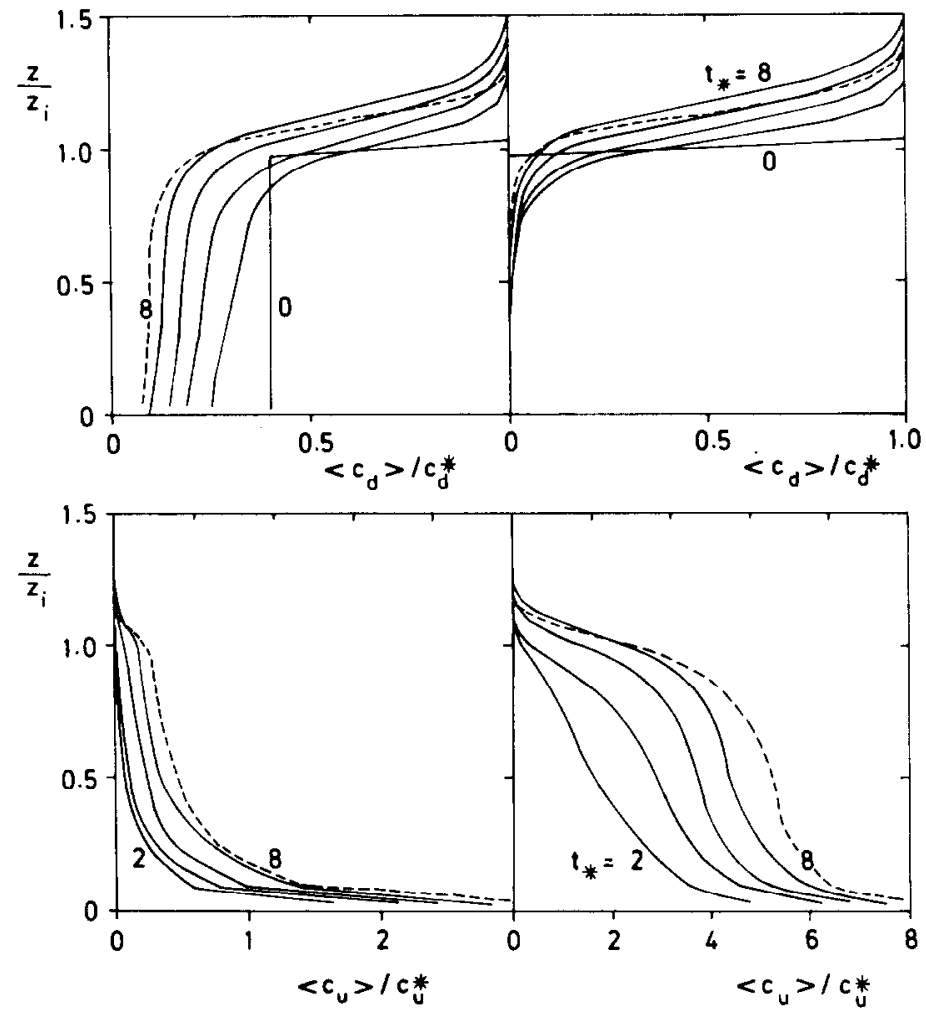

Fig. 8. Mean concentration profiles vs normalized height at a sequence of nondimensional times for top-down and bottom-up diffusing components with finite reaction rate $(R=1, n=20)$. Full curves: grid $\mathrm{C}$; dashed curves: grid $\mathrm{M}$ at time $t_{*}=8$. Left panels: $c_{\mathfrak{d}}^{0} / c_{d}^{*}=0.4$; right panels: $c_{\mathfrak{d}}^{0}=0$.

the emitted quantity fills up the mixed layer quickly in the unmixed case.

For traditional turbulence modelling, the effective eddy diffusivities shown in Fig. 9 are of interest. As Pyle and Rogers (1980), in the case of stratospheric planetary waves, we find that the eddy diffusivities differ for various reacting components. Although the order of magnitude of the eddy diffusivities is unchanged, the profiles are very different from those shown in Fig. 4. This is to be expected from the 


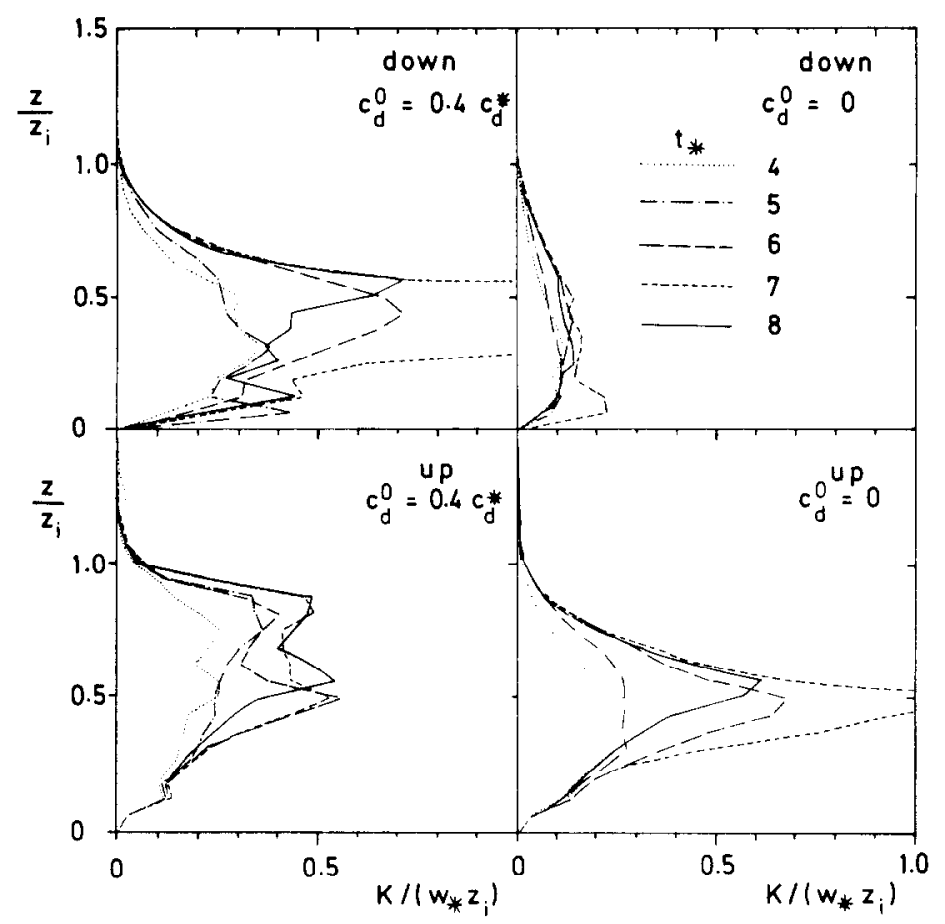

Fig. 9. Effective eddy-diffusivities $K_{u}$ and $K_{d}$ vs height at a sequence of nondimensional times. $R=1, n=20$, grid $C$. Left panels: $c_{d}^{0} / c_{d}^{*}=0.4$; right panels: $c_{d}^{0}=0$.

second-order equations (Murthy, 1975) because they show that the fluxes are determined not only by concentration gradients but also by the chemical reactions (and the vertical heat flux). Thus the eddy diffusivities must change. The results for the mixed and unmixed case differ strongly. The eddy diffusivities are increased if the gradient of the concentration gets larger importance than other terms contributing to the fluxes. This is true in particular for the downward component in the mixed case. Hence, although the chemical reactions have no direct effect on the turbulent flow field in these cases, it does have large effects on the turbulent transport properties if measured in terms of eddy diffusivities. Of course, this just illustrates the fundamental weakness of the eddy diffusivity concept.

The upper panels of Fig. 10 show the mean reaction rates as computed from the LES and the lower panels the result which one would obtain from the mean profiles of the LES-results. The actual reaction rates are smaller than suggested by the mean profiles and thus indicate that the two components are more or less segregated in updrafts and downdrafts. This segregation is typical for such mixing layers and has been found also by Riley et al. (1986). The resultant reaction rate is small because it requires smaller-scale mixing between the updrafts and downdrafts. The reaction rate differs by up to about $50 \%$ from that suggested by the mean profiles and this difference is particularly large in absolute values for the unmixed case. We note that a local maximum of reaction rate occurs in both the mixed and unmixed cases near the inversion. This originates from strong updrafts which succeed in reaching the inversion without strong depletion of the emitted component; there this component comes into close and long duration contact with the other component from above the inversion. Thus the turbulence has a strong effect on chemistry in this case.

Figure 11 shows Fourier cospectra of the concentrations, and shows spectra of the vertical flux of the upward diffusing component. (Flux-spectra of the downward diffusing component are not shown because its qualitative structure equals that of the upward flux spectrum, but with negative sign and smaller magnitude.) These one-dimensional spectra were obtained by Fourier transforming the fields along horizontal lines and averaging over all parallel lines at fixed height and time. Similar spectra for the velocity components and the vertical heat flux have been reported by Schmidt and Schumann (1989). Note that the spectra are multiplied with the horizontal wavenumber before plotting so that the contributions from high wave-numbers appear exaggerated. The spectral values are small at high wave-numbers everywhere except at the lowest level where such small-scale contributions are to be expected. With the exception of the flux-spectrum near the surface, the spectra for $R=1$ decay to zero with increasing wave number more quickly than for $R=0$ so that the small-scale contributions for $R=1$ are relatively less important than for $R=0$. However, for very rapid chemical reactions (not plotted) the relative importance of small-scale contri- 


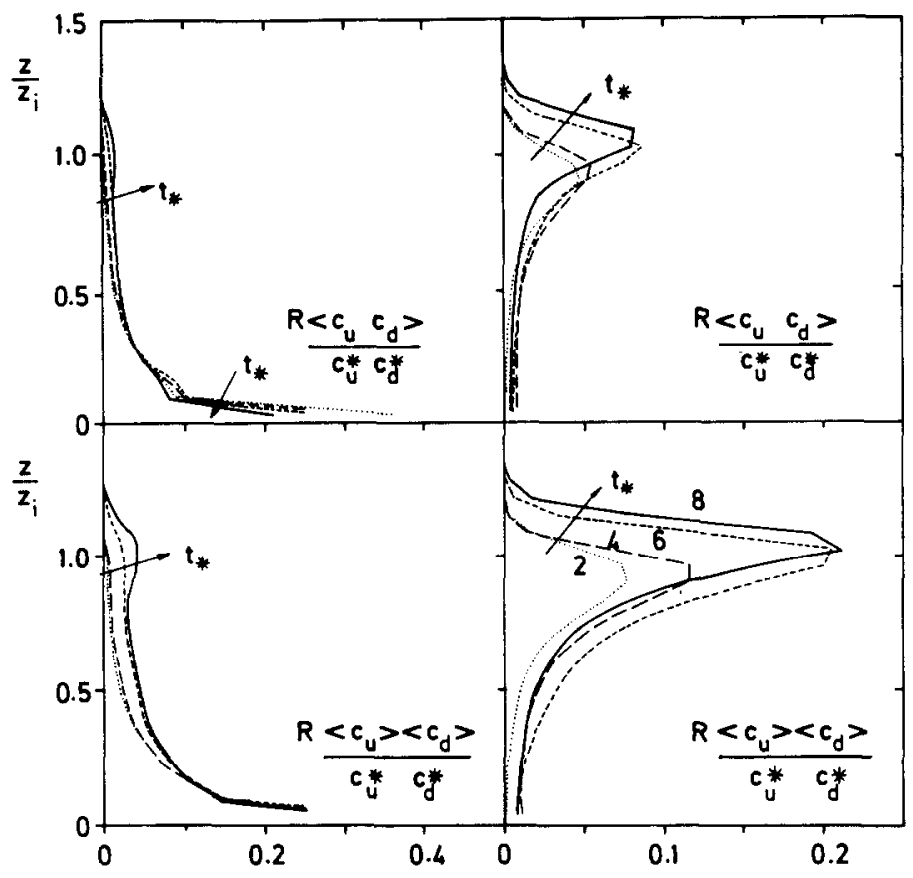

Fig. 10. Normalized reaction rates $R\left\langle\bar{c}_{u} \bar{c}_{d}\right\rangle /\left(c_{u}^{*} c_{d}^{*}\right)$ (top panels) and corresponding products of the mean values $R\left\langle\bar{c}_{u}\right\rangle\left\langle\bar{c}_{d}\right\rangle /\left(c_{u}^{*} c_{d}^{*}\right)$ (bottom panels) vs height. $R=1, n=20$, grid C. Left panels: $c_{d}^{0} / c_{d}^{*}=0.4$; right panels: $c_{d}^{0}=0$.
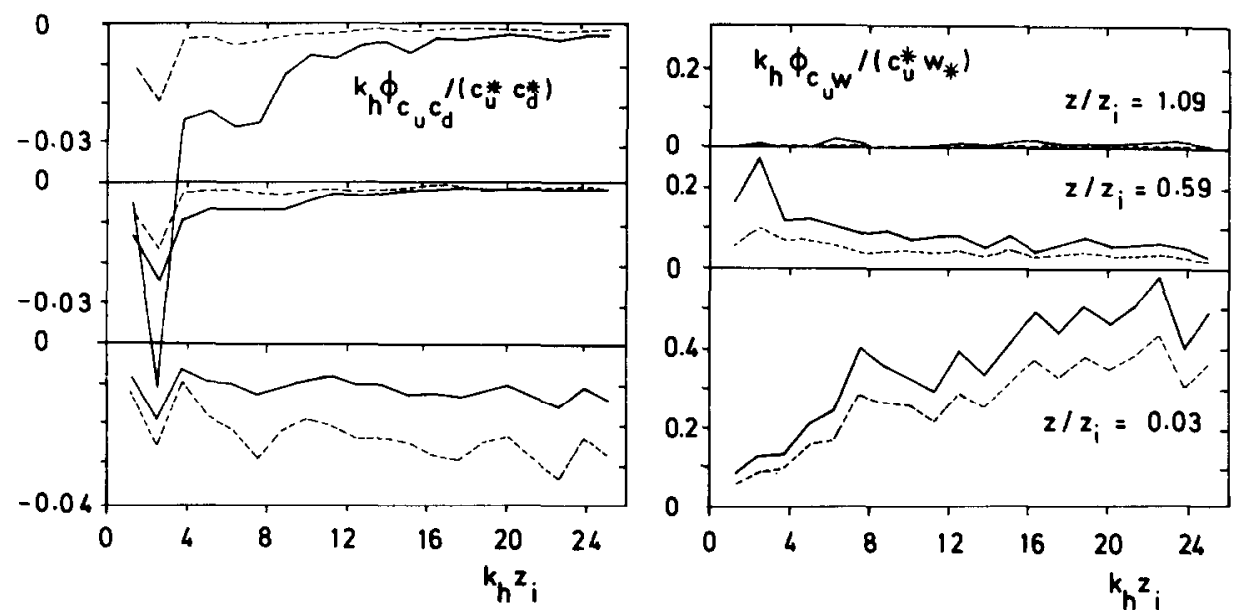

Fig. 11. Normalized horizontal cospectra $\Phi$ of $\bar{c}_{u} \bar{c}_{d}$ (left) and $\overline{c_{u}} \bar{w}$ (right) vs normalized wave number $k_{k} z_{i}$; $t_{*}=8$; grid C. Dashed curves: $R=0$; full curves: $R=1$. The lower, middle and upper panels apply to $z / z_{i}=0.03,0.59,1.09$, respectively.

butions get enhanced. On the other hand, the absolute magnitude of the spectra at high wave numbers is largest for $R$ of the order unity so that this is the most demanding case with respect to resolution. Near the surface, the spectral values at non-dimensional wave number 8 , i.e. wavelength $0.8 z_{i}$, shows the importance of contributions which are likely to be related to the distance between updrafts and downdrafts. In general, however, contributions at a non-dimensional wave number of about 3 (length-scale $2 \pi / k_{h} \cong 2 z_{i}$ ) dominate. This corresponds to the mean distance between updrafts. Note that these cospectra were obtained from the simulations with the coarsest grid. We conclude that even for such grids the most important spectral components are resolved. The (unnormalized) spectra generally decay more rapidly than $k_{h}^{-1}$ and, hence, subgrid-scale contributions to the chemical reactions will not be of large importance for the present study.

Further results (not plotted) show that the spectra for $R=0$ and 0.1 typically differ by only $10 \%$ in magnitude. Thus, the case $R=0.1$ corresponds to slow 


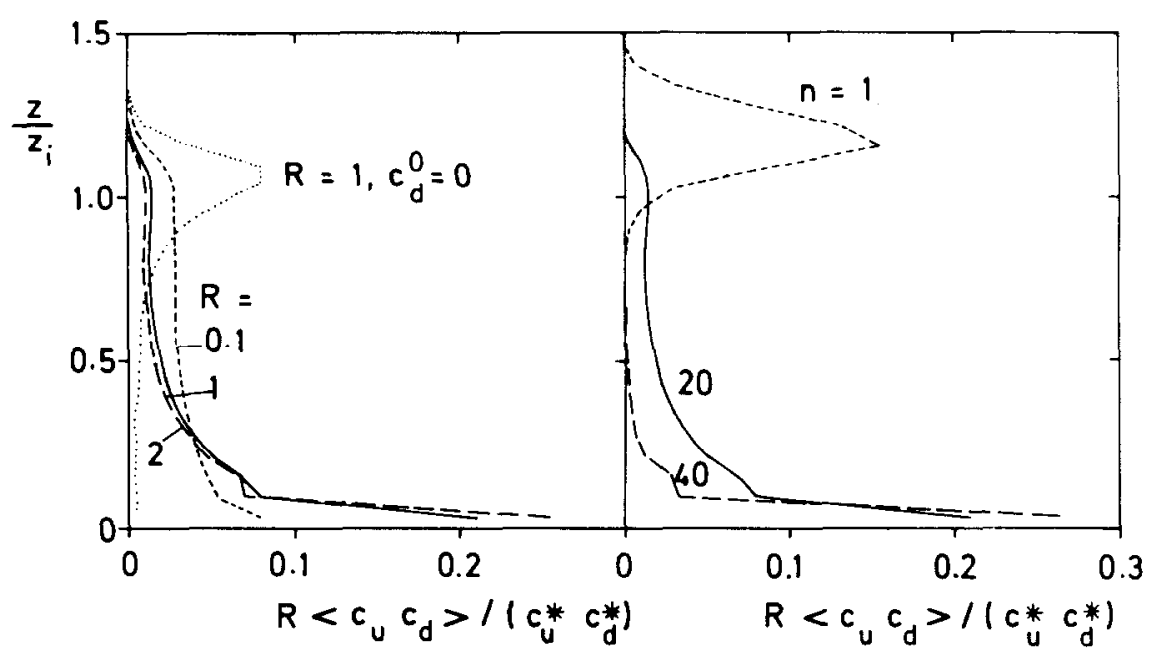

Fig. 12. Normalized reaction rates $R\left\langle\bar{c}_{u} \bar{c}_{d}\right\rangle /\left(c_{u}^{*} c_{d}^{*}\right)$ at time $t_{*}=8$ (grid C). All cases except one (dotted) apply to $c_{\mathrm{d}}^{0}=0.4 c_{d}^{*}$. Left: for $n=20$, and various values of $R$; right: $R=1$, and $n=1,20,40$.

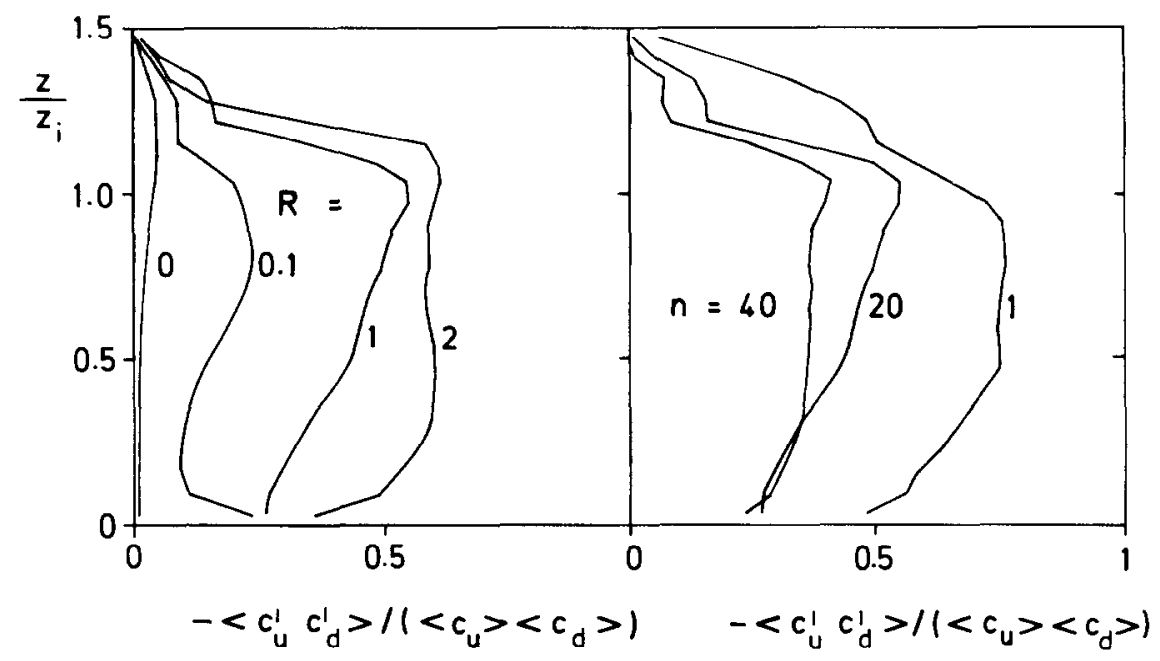

Fig. 13. Segregation coefficient $-\left\langle c_{u}^{\prime} c_{d}^{\prime}\right\rangle /\left(\left\langle c_{u}\right\rangle\left\langle c_{d}\right\rangle\right)$ at time $t_{*}=8 ; c_{d}^{0}=0.4 c_{d}^{*}$, grid C. Left: $n=20$, right: $R=1$

chemistry which is not significantly limited by turbulent transport. Also, cases $R=1$ and 2 show very similar spectra, so that $R=1$ already belongs to a case with rapid chemistry limited by turbulent diffusion. For infinitely rapid chemical reactions, the reaction zone becomes very small and thus the cospectra of $c_{u} c_{d}$ will be dominated by small-scale contributions everywhere. The cases considered here are still far from this limiting case.

\section{Parameter variations}

Figure 12 shows the effect of the parameters $R$ and $n$ on the normalized reaction rate profiles at the final time of the LES. With one exception, these results apply to the mixed cases. The results for $R=1$ and 2 are very similar indicating convergence toward the diffusive limit for $R>1$. For $R=0.1$ the reaction rate is closer to a constant value within the mixed layer. For this rather slow reaction rate, turbulence succeeds in providing fairly complete mixing before the reaction takes place. In the unmixed case, the reaction rate is maximum near the inversion while it is greatest near the surface in the mixed case, as explained above. The results for fixed $R$ but varying concentration ratios show that for a very small fraction of downward diffusing component $(n=1)$ the reaction dominates near the inversion and is virtually zero at small heights because the component $c_{d}$ gets depleted before downdrafts reach the surface. Conversely, large values of $n$ confine the reaction to a very narrow zone at the surface. This was concluded already from the considerations for the infinite reaction case.

Figure 13 shows the so-called 'segregation coefficient'. It measures the relative deviation of the actual 
reaction from that value suggested by the mean concentration profiles. The value is negative. This can be understood from Equation (6). In the mixing region of two components the (pasitive) products of fluxes and gradients of the two components cause negative trends of the correlation between the two concentrations even without reactions, and this is reflected in the result for case $R=0$. However, this effect is not very large for the present conditions because the gradients are small where the fluxes are large and vice versa. Strong negative trends arise from the products of concentration variances with mean concentrations for $k>0$. The resultant segregation coefficients have maximum magnitude at heights where the actual reaction rate is small, because large negative values of the covariances counteract the negative trends. Figure 13 shows also the results for $R=1$ and $n=1,20$, 40 . For large values of $n$, the segregation coefficient is reduced because of very small concentration variances outside the reaction zone near the surface.

\section{COMPARISON WITH A BOX-MODEL}

In this section, we compare the LES with the boxmodel described in the Appendix. Figure 14 shows results for the time evolution of mixed-layer mean values of the concentrations, as obtained by averaging the LES-results from the surface up to $0.8 z_{i}$. The results show the trends as discussed before. In most cases, the predictions of the box-model, Equations (13) and (14), compare with the LES-model fairly well. For
$R=0$, the concentration $c_{d}$ increases in the LES but decreases in the box-model. This shows that Equation (15) slightly underestimates the actual entrainment rate. For $R=0.1$, the quantitative differences are not much larger. This is true even for infinite reaction rate, although the box-model, Equations (20) and (21), cannot describe the coexistence of both components in the mixed layer. Essential quantitative differences occur only for $R=1$. Further evaluations (not plotted) exhibit increasing differences for increasing finite values of $R$ and decreasing values of $n$. Also, the differences are larger in the unmixed case $\left(c_{d}^{0}=0\right)$ than in the mixed case shown here. The box-model overestimates the decay rate from chemical reactions because it neglects spatial segregation of the reactants.

\section{CONCLUSIONS}

By means of large-eddy simulations of the CBL, the effect of turbulence on chemical reactions and the dependence of effective diffusivities on chemical reactions has been investigated for idealized conditions.

Without chemical reactions, the CBL transports tracers upwards more strongly than downwards. As an alternative to Wyngaard's (1987) suggestion we explain this difference to be caused by the different mixing of components into high speed updrafts and slow downdrafts, and by the combined forcing from concentration gradients and buoyancy, which act together for upward transport but are counteracting for downward motion.

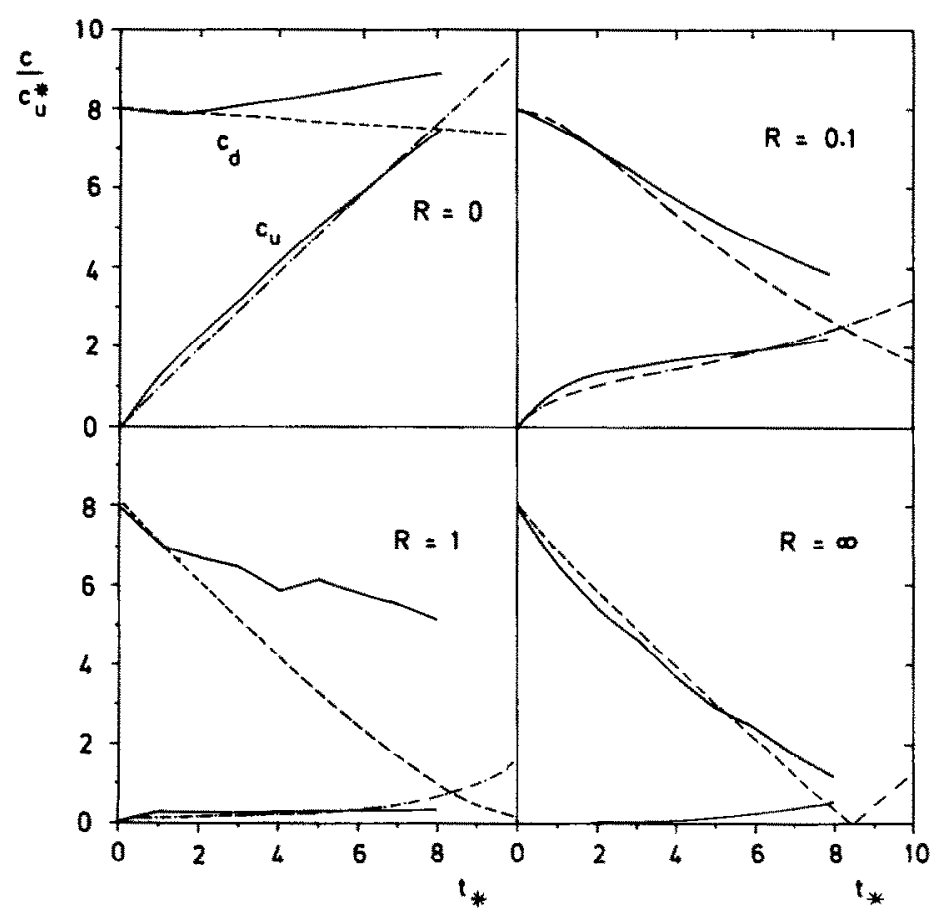

Fig. 14. Mixed-layer averaged concentrations $c_{u}$ and $c_{d}$ vs non-dimensional time from the LES (full curves) and from the box-model (dashed and dash-dotted curves), for various values of $R$, and $n=20, c_{d}^{0}=0.4 c_{u}^{*}$, grid $C$. 
For cases with chemical reactions of a simple binary irreversible kind, the results depend on the parameters $R, n$ and the initial conditions. Values of the order $R=1, n=20$, are characteristic in magnitude for reactions between $\mathrm{O}_{3}$ and $\mathrm{NO}$ in a fully developed CBL. The reaction zone is very thin for large values of $R$. The reaction maximum occurs at the lower surface for large values of the downward diffusing component and coincides with the interfacial layer at the top of the CBL if the upward component dominates in concentration magnitude. The reaction is limited by turbulence for large values of $R$. We found that a value of $R>0.1$ must be classified as being large and a value of $R \leqslant 0.1$ as small in this sense. The segregation coefficient exceeds 0.3 for $R>0.1$. The effective eddy diffusivities may be either enhanced or reduced in the presence of chemical reactions although the order of magnitude of eddy diffusivities remains unchanged. However, results (not shown here) of a simple onedimensional diffusion model give concentration profiles which differ essentially from the LES results even if Equation (10) is used. Hence, eddy-diffusivity models suffer from scverc limitations. A simple boxmodel might often be of practical value, at least if $R \leqslant 0.1$ or $R \gg 1$. It requires, however, precise knowledge of the entrainment velocity $w_{\mathrm{e}}$ for which Equation (15) gives only a rough approximation. For the reactive case, second-order models at least help in understanding of the trends induced by various forcings.

Results obtained from grid $\mathrm{C}$ and $\mathrm{M}$ show that the coarse grid provides the minimum resolution necessary for such studies. For $R \leqslant 2$, it has been demonstrated by spectral analysis that the most important flux and reaction contributions are resolved by the LES, even for grid C, so that the neglect of chemical effects on the subgrid-scale contributions is justified. A value of $R$ of order unity is most demanding with respect to grid resolution. In the limit to infinite $R$, the LES is not seriously deteriorated by the unresolved contributions because in most parts the concentrations behave like a conserved passive scalar.

The present study shows that much insight into the dynamics of turbulent reacting flows can be obtained from LES. However, a more realistic chemistry model has yet to be included to compare the results with observations. In spite of the large computational effort which such extended models require, such studies appear to be feasible in the near future.

Acknowledgement-This study initiated from a stimulating discussion with Dr D. Ehhalt. I thank Drs A. Ebel, E. Ebert, H. Schmidt and R. B. Stull for helpful comments and Mrs J. Freund for plotting the results.

\section{REFERENCES}

Chang J. S., Brost R. A., Isaksen I. S. A., Madronich S., Middleton P., Stockwell W. R. and Walcek C. J. (1987) A three-dimensional Eulerian acid deposition model: physi- cal concepts and formulation. J. geophys. Res. 92, $14,681-14,700$.

Chatfield R. B. and Brost R. A. (1987) A two-stream model of the vertical transport of trace species in the convective boundary layer. J. geophys. Res. 92, 13,263-13,276.

Deardorff J. W. (1974) Three-dimensional numerical study of turbulence in an entraining mixed layer. Boundary-Layer Met. 7, 199-226.

Ebert E. E., Schumann U. and Stull R. B. (1989) Nonlocal turbulent mixing in the convective boundary layer evaluated from large-eddy simulation. J. atmos. Sci. 46, (in press).

Georgopoulos P. G. and Seinfeld J. H. (1986) Mathematical modeling of turbulent reacting plumes-I. General theory and model formulation. Atmospheric Environment 20, 1791-1807.

Herring J. R. and Wyngaard J. C. (1987) Convection with a first-order chemically reactive passive scalar. In Turbulent Shear Flows 5 (edited by F. Durst et al.), pp. 324-336. Springer, Berlin.

Lamb R. G. and Durran D. R. (1979) Eddy diffusivities derived from a numerical model of the convective planetary boundary layer. II Nuovo Cimento 1C, 1-17.

Launder B. E. (1975) On the effects of a gravitational field on the turbulent transport of heat and momentum. J. Fluid Mech. 67, 569-581.

Lenschow D. H., Delany A. C., Stankov B. B. and Stedman D. H. (1980) Airborne measurements of the vertical flux of ozone in the boundary layer. Boundary-Layer Met. 19, 249-265.

Leonard A. D. and Hill J. C. (1988) Direct numerical simulation of turbulent flows with chemical reaction. J. Sci. Comput. 3, 25-43.

Libby P. A. and Williams F. A. (1980) Turbulent Reacting Flows, p. 243. Springer, Berlin.

Moeng C.-H. and Wyngaard J. C. (1984) Statistics of conservative scalars in the convective boundary layer. $J$. atmos. Sci. 41, 3161-3169.

Murthy S. N. B. (ed.) (1975) Turbulent Mixing in Nonreactive and Reactive Flows, pp. 1-84. Plenum Press, New York.

O'Brien E. E. (1971) Turbulent mixing of two rapidly reacting chemical species. Phys. Fluids 14, 1326-1331.

Pyle J. A. and Rogers C. F. (1980) Stratospheric transport by stationary planetary waves-the importance of chemical processes. $Q$. $J l R$. met. Soc. 106, 421-446.

Riley J. J., Metcalfe R. W. and Orszag S. A. (1986) Direct numerical simulations of chemically reacting turbulent mixing layers. Phys. Fluids 29, 406-422.

Schmidt H. (1988) Grobstruktur-Simulation konvektiver Grenzschichten. Thesis Univ. München, report DFVLRFB 88-30, DLR, Postfach 906058, D-5000 Köln 90.

Schmidt H. and Schumann U. (1989) Coherent structure of the convective boundary layer derived from large-eddy simulations. J. Fluid Mech. 200, 511-562.

Schumann U. (1988) Minimum friction velocity and heat transfer in the rough surface layer of a convective boundary layer. Boundary-Layer Met. 44, 311-326.

Schumann U., Hauf T., Höller H., Schmidt H. and Volkert H. (1987) A mesoscale model for the simulation of turbulence, clouds and flow over mountains: formulation and validation examples. Beitr. Phys. Atmosph. 60, 413-446.

Seinfeld J. H. (1986) Atmospheric Chemistry and Physics of Air Pollution, p. 738. Wiley, New York.

Stull R. B. (1988) An Introduction to Boundary Layer Meteorology, p. 666. Kluwer, Dordrecht.

Sun W.-Y. (1986) Air pollution in a convective boundary layer. Atmospheric Environment 20, 1877-1886.

Wyngaard J. C. (1987) A physical mechanism for the asymmetry in top-down and bottom-up diffusion. J. atmos. Sci. 44, 1083-1087.

Wyngaard J. C. and Brost R. A. (1984) Top-down and bottom-up diffusion of a scalar in the convective boundary layer. J. atmos. Sci. 41, 102-112. 
Table 1. Steady-state values for bottom-up concentration $c_{u}$ and top-down concentration $c_{d}$ in the boxmodel as a function of reactivity parameter $R$ and concentration ratio $n=c_{d}^{*} / c_{u}^{*}$

\begin{tabular}{lcccccccc}
\hline$R$ & 0 & 0.1 & 1 & 2 & 1 & 1 & 1 & 1 \\
$n$ & 20 & 20 & 20 & 20 & 1 & 40 & 100 & 200 \\
\hline$c_{u}$ & 117.6 & 97.7 & 97.6 & 97.6 & 116.8 & 77.7 & 17.8 & 0.0428 \\
$c_{d}$ & 5.66 & 0.0173 & $1.74 \mathrm{E}-3$ & $8.71 \mathrm{E}-4$ & $6.10 \mathrm{E}-5$ & $4.38 \mathrm{E}-3$ & 0.0476 & 23.3 \\
\hline
\end{tabular}

\section{APPENDIX: BOX-MODEL AND STEADY-STATE SOLUTION}

Here we set up the equations which apply if the concentrations $c_{u}$ and $c_{d}$ in the mixed layer are approximately homogeneous due to rapid turbulent mixing. This results in the socalled 'box-model'. First we consider the case of zero or finite reaction rate; at the end of this Appendix, the case of infinite reaction rate will be discussed.

The box extends from the surface up to the inversion at height $z_{i}$. It receives material $c_{u}$ from below due to the surface flux $w_{*} c_{u}^{*}$ and loses material by the vertically integrated chemical reaction rate $k z_{i} c_{u} c_{d}$. Moreover, it is diluted due to entrainment of 'clean air' from above the inversion with the flux $w_{\mathrm{e}} c_{\mu}$. Here $w_{\mathrm{e}}$ is the entrainment velocity. Likewise, material $c_{d}$ enters the box from above the inversion with a flux $w_{\mathrm{e}}\left(c_{d}^{*}-c_{d}\right)$, is deposited at the surface with a flux $w_{d} c_{d}$, and is lost at the same chemical reaction rate. Thus, the boxintegrated concentrations satisfy

$$
\begin{aligned}
& \frac{\mathrm{d}\left(z_{i} c_{u}\right)}{\mathrm{d} t}=w_{*} c_{u}^{*}-c_{u}\left(w_{\mathrm{e}}+k z_{i} c_{d}\right), \\
& \frac{\mathrm{d}\left(z_{i} c_{d}\right)}{\mathrm{d} t}=w_{\mathrm{e}}\left(c_{d}^{*}-c_{d}\right)-c_{d}\left(w_{d}+k z_{i} c_{u}\right) .
\end{aligned}
$$

The entrainment velocity may be approximated by the 'encroachment velocity', $w_{\mathrm{e}}=\mathrm{d} z_{i} / \mathrm{d} t=(\partial\langle T\rangle / \partial z)^{-1} Q_{s} / z_{i}$ (Stull, 1988, p. 455), so that

$$
w_{\mathrm{c}}=\mathrm{Fr}^{2} w_{*},
$$

where $\mathrm{Fr}=\boldsymbol{w}_{*} /(\mathrm{Nz})$ is the convective Froude number. This gives a lower bound to the entrainment velocity because the actual rise of the CBL will be typically 10-20\% larger due to additional warming of the $\mathrm{CBL}$ by turbulent entrainment. For vanishing mean wind, the deposition velocity can be estimated by analogy to the heat transfer rate deduced in Schumann (1988). For zero surface concentration, it results in

$$
w_{d}=w_{*}\left(10 z_{i} / z_{0}\right)^{-1 / 3},
$$

where $z_{0}$ is the effective roughness length (which is assumed to be the same for momentum and material transport for simplicity).

For quasi stcady statc (constant $z_{i}$ ) the solutions to Equations (13) and (14) are

$$
\begin{aligned}
& c_{u}=w_{*} c_{u}^{*}, c_{\mathrm{e}}+k z_{i} c_{d} \\
& w_{d}-B+\sqrt{\left(B^{2}+A\right)}, \\
& B=\frac{w_{\mathrm{e}}\left(w_{d}+w_{\mathrm{e}}\right)+k z_{i}\left(c_{u}^{*} w_{*}-c_{d}^{*} w_{\mathrm{e}}\right)}{2 k z_{i}\left(w_{\mathrm{e}}+w_{d}\right)}, A=\frac{w_{\mathrm{e}}^{2} c_{d}^{*}}{k z_{i}\left(w_{\mathrm{e}}+w_{d}\right)},
\end{aligned}
$$

for $k>0$, and

$$
c_{u}=\frac{w_{*}}{w_{\mathrm{e}}} c_{u}^{*}, c_{d}=\frac{w_{\mathrm{e}}}{w_{\mathrm{e}}+w_{d}} c_{d}^{*}
$$

for $k=0$. In Table 1, the steady-state box-solutions are given for the parameter values used in the LES. Additional entries are given for large values of the concentration ratio $n=c_{d}^{*} / c_{u}^{*}$. We see that the steady-state concentration values for $c_{u}$ are very large if $k$ is small and $n<100$. The concentration results depend mainly on $n$ while the value of $R$ is important only if both concentrations are of comparable magnitude. If $n$ is less than about 100 , the bottom-up concentration dominates because its surface flux is large in comparison to the entrainment flux of $c_{d}$. The large amount of component emitted at the surface consumes most of the entrained component by the chemical reaction.

Without chemical reactions and entrainment, the bottomup concentration increases linearly by one unit of $c_{u}^{*}$ per time unit $z_{i} / w_{*}$. Obviously, the steady-state values are of order $w_{*} / w_{\mathrm{e}}$. Hence, it takes a time of order $z_{i} / w_{\mathrm{e}}=\mathrm{Fr}^{-2} z_{i} / w_{*}$ to reach the steady state if the reaction rate is small. This time is much larger than the time-scale of convection in the CBL because $\mathrm{Fr}^{-2}=118$ in this case. Since the time-scale of convection amounts to about $18 \mathrm{~min}$, such a steady-state cannot be achieved within a day.

In the case of infinite reaction rate $(k \sim R \rightarrow \infty)$, either $c_{u}$ or $c_{d}$ gets quickly depleted from the mixed layer by the rapid reaction. The non-zero component follows an equation similar to those given in (13) and (14) but with the reaction rate replaced by the flux of the smaller component into the box

$$
\begin{aligned}
& \frac{\mathrm{d}\left(z_{i} c_{u}\right)}{\mathrm{d} t}=w_{*} c_{u}^{*}-w_{e}\left(c_{u}+c_{d}^{*}\right), c_{d}=0, \\
& \frac{\mathrm{d}\left(z_{i} c_{d}\right)}{\mathrm{d} t}=w_{\mathrm{e}}\left(c_{d}^{*}-c_{d}\right)-c_{d} w_{d}-w_{*} c_{u}^{*}, c_{u}=0 .
\end{aligned}
$$

The steady state solutions are

$$
\begin{aligned}
& c_{u}=\frac{w_{*} c_{u}^{*}-w_{\mathrm{e}} c_{d}^{*}}{w_{\mathrm{e}}}, c_{d}=0, \text { if } n=\frac{c_{d}^{*}}{c_{\mathrm{u}}^{*}} \leqslant \frac{w_{*}}{w_{\mathrm{e}}} \cong F r^{-2}, \\
& c_{d}=\frac{w_{\mathrm{c}} c_{d}^{*}-w_{*} c_{u}^{*}}{w_{e}+w_{d}}, c_{\mathrm{u}}=0, \text { if } n>\frac{w_{*}}{w_{\mathrm{r}}} .
\end{aligned}
$$

Again, the time required to reach these steady-state values is large and of the order $z_{i} / w_{\mathrm{e}}$. 